\title{
An overview of the RFCS project V\&V framework: optimization-based and linear tools for worst-case search
}

\author{
A. Marcos - P. Rosa - C. Roux - M. \\ Bartolini · S. Bennani
}

Received: date / Accepted: date

\begin{abstract}
This article presents the application of nonlinear (simulation-based) and linear (structured singular value) worst-case tools to the VEGA launcher Verification and Validation (VEV) process, during atmospheric ascent. The simulation-based worst-case evaluation is performed by minimizing a set of cost functions that capture launcher performance objectives, using the WorstCase Analysis Optimization Tool (WCAT) and a high-fidelity nonlinear simulator of VEGA. The linear worst-case search uses the structured singular value $(\mu)$ and a linear fractional transformation (LFT) model representing the yaw rigid-motion of the VEGA launcher but numerically evaluated using time simulation data from the VEGA simulator. In order to facilitate the analysis of the worst-case results as well as the comparison between the two analysis tools, a selection of the most critical uncertainties is performed using sensitivity analysis based on selected nonlinear simulator time responses. It is highlighted that the presented analysis tools are complementary to traditional Monte-Carlo approaches in that they strive to identify worst-case uncertainty combinations as opposed to providing probabilistic guarantees on performance metric satisfaction. In addition, as it will be shown, these approaches require only a fraction of the time required to perform a Monte Carlo campaign.
\end{abstract}

\footnotetext{
A. Marcos

Deimos Space S.L.U., Madrid, 28760, Spain

Present address: University of Bristol, Aerospace Engineering Department, Bristol, BS8

1TR, United Kingdom

E-mail: andres.marcos@bristol.ac.uk

P. Rosa

Deimos Engenharia, Lisbon, 1998-023, Portugal

C. Roux, M. Bartolini

ELV, Rome, 00034, Italy

S. Bennani

ESA_ESTEC, Noordwijk, 2201AZ, The Netherlands
} 


\section{Introduction}

The state-of-practice for launcher Verification \& Validation (V\&V) [15] entails analysis of frequency-domain requirements (such as gain and phase margins) through a set of predefined vertex cases as well as analysis of time-domain requirements (such as thruster fuel consumption and maximum aero-loads) through nonlinear simulations. The latter is typically performed in a MonteCarlo (MC) setting (i.e. probabilistic approach) and/or using a selected set of vertex or worst-cases (i.e. deterministic approach).

Traditional MC campaigns consist of randomly sampling the uncertain parameters according to statistical distributions and deducing the values of criteria involved in the requirements. The number of simulations depends on specified probability and confidence levels [6]. The MC approach is the standard in industrial $\mathrm{V} \& \mathrm{~V}$ due to its probabilistic guarantees and the fact that it can be applied to any type of simulation model. Nonetheless, due to the first advantage it requires a high number of sampled simulations (i.e. in the thousands, or even hundreds of thousands for highly critical systems) and the results come back without guaranteed proofs for the full parameter space. Similarly, vertex approaches whereby the corner cases (minimum and maximum along each uncertain parameter direction) are checked, are unfeasible whenever the dimension of the problem is relatively high. This is compensated by introducing engineering knowledge on the system which helps limit the number of corner cases to be examined. Nonetheless, the analysis is very limited and obviates altogether parameter combinations away from the extremes.

To overcome these limitations, many advances have been explored in the field of aeronautical V\&V (cf. [5, 3, 8]). One of the venues explored, which has proved very successful, has been the use of advanced optimization-based worstcase search algorithms (see, for instance, $[17,16,13,14]$ ). The main downside of these approaches is the lack of guarantees in finding the worst-case. However, these methods typically can identify performance violation cases quickly and their usage requires only minimal adaptation (of the typical simulation models used in industry, e.g. Simulink/Matlab, Fortran, C-code). The other venue that has been followed in order to provide analytical guarantees is the use of analytical approaches such as the structured singular value $\mu$ (see $[1,2,14]$ ). The downsides of $\mu$-analysis are its linear nature, exemplified by its reliance on linear fractional transformation (LFT) models typically arising from linear dynamic systems, and the lack of associated probabilities to the identified worst-cases, which are necessary to provide a quantitative risk value. The advantages are that the worst-case identified typically carry over to the nonlinear system (if the LFT model properly captures its dynamic behavior) and that it provides analytically guaranteed bounds on the robust stability and performance degradation.

In this article, the results from the application of the above two worst-case search techniques to the VEGA launcher system during the atmospheric ascent phase (P80) are presented. This was performed within a European Space Agency funded project entitled "Robust Flight Control System Design Verifi- 
cation and Validation Framework (RFCS)". The presented work is an extension of the results presented in $[13,14]$ using an enhanced VEGA nonlinear simulator, the newest $3^{\text {rd }}$ flight qualification mission data and controller, and more detailed $\mu$ analyses (including specific gain/phase margin uncertainty). It is shown that both, the simulation and the linear worst-case approaches, are able to successfully identify numerous performance-violating cases - valid not only in the nonlinear simulator used for verification but also in the official VEGA simulator used for validation. Assessment of these cases by the GNC team at ELV, the Italian prime contractor for VEGA, validated the cases but ruled them out as with very low probability of occurrence. Indeed, the VEGA launcher has now successfully performed four qualification flights.

The organization of the paper is as follows: Section 2 briefly describes the VEGA launcher and the main characteristics of the nonlinear simulators. Section 3 presents the simulation-based worst-case search methodology used, including the numerical sensitivity analysis performed on the defined cost functions and the selection of the worst-case parameter sets to be investigated. It follows Section 4, where the general ideas behind $\mu$ analysis and LFT modelling are presented, including the description of the linear launcher model used for the latter and the overall analysis methodology followed. The results obtained with each of these two worst-case approaches are provided and discussed in Section 5 with some concluding remarks given in Section 6 .

\section{VEGA Launcher \& Nonlinear Simulators}

VEGA is the new European Small Launch Vehicle developed under the responsibility of ESA. The prime contractor for the launch vehicle is ELV (Italy). The launcher successfully performed a maiden launch at the beginning of 2012 from the Centre Spatial Guyanais in Kourou, a $2^{\text {nd }}$ flight in May 2013, a $3^{\text {rd }}$ flight in April 2014, and more recently the $4^{\text {th }}$ qualification flight on February 2015 .

VEGA follows a four-stage approach formed by 3 solid propellant motors (P80, Zefiro 23 and Zefiro 9) providing thrust for the $1^{\text {st }}, 2^{\text {nd }}$ and $3^{\text {rd }}$ stages; and, a bi-propellant liquid engine (LPS) on the $4^{\text {th }}$ stage. All four stages are controlled via a thrust vectoring system (TVC). There is also a Roll and Attitude Control System (RACS) performing 3-axes control during the ballistic phase and roll rate control during the propelled phases.

The official non-real time high-fidelity nonlinear simulator used in the VEGA program is called VEGAMATH. This simulator is characterized by:

- High-fidelity 6 Degrees-of-Freedom motion

- Tail-Wag-Dog effects

- Bending and sloshing modes

- Full external environment (rotating Earth, winds, gusts ...)

- Disturbances (torques at separation, bias, offsets)

- Nonlinear aerodynamics (including aero-elastic effects)

- TVC system (including computing delays, backlash, bias ...) 
- Full representative code implementing the actual Guidance, navigation and control (GNC) system

- Propulsion and mass-center-inertia (MCI) properties

- Detailed inertial navigation system (INS)

- Detailed RACS models (thermal/thrust dynamics, filters, quantization ...)

The simulator used by the RFCS consortium is called VEGACONTROL and is based on VEGAMATH but tailored to simulate the $1^{\text {st }}$ atmospheric phase (i.e. P80). The VEGACONTROL simulator requires on average 3.3 seconds per simulation run, in a PC with an Intel Core i5 CPU @ $3.20 \mathrm{GHz}$. This is several orders of magnitude faster than VEGAMATH (the exact running time for the latter cannot be provided due to proprietary concerns). In comparison with [14], the VEGACONTROL version used in this work includes enhanced correlation of key uncertain parameters, bending and sloshing modes and wind-scaling profiles. Note that the development of the LFT model, used for the worst-case $\mu$ analysis in Section 4 , is based only on the yaw rigid-motion of the vehicle (i.e. no direct flexible, sloshing or wind effects are introduced in the LFT model, although the data used for its numerical evaluation comes from the enhanced VEGACONTROL and thus contains these effects). Further, it is highlighted that for all the worst-cases obtained using both methods, the RFCS consortium used VEGACONTROL to verify the cases but then these were validated by ELV on VEGAMATH.

Both VEGACONTROL and VEGAMATH allow modifying the scattering values (uncertainties and dispersions) of up to 125 different uncertain parameters organized into 9 sets (aeroelasticity, aerodynamics, wind, IRS, thrust, MCI, thrust offset \& misalignment, atmosphere, separation disturbance). Each scattering variable is represented by a "flag" parameter with values ranging in the interval $[-1,1]$, with the zero value indicating "nominal".

\section{Simulation-based WCC Search Methods}

In reference [13] a worst-case time-domain optimization toolbox known as "Worst-Case Analysis Toolbox (WCAT)", developed in previous ESA projects [16], was used to identify worst-cases using the mission trajectory and controller for the $2^{\text {nd }}$ VEGA qualifying flight (as well as a version of VEGACONTROL without the aforementioned effects). Using in an iterative manner a number of pre-defined parameters sets, 58 cases that violated one or several performance bounds were identified and verified in the VEGACONTROL and validated in VEGAMATH.

In this present work, the $3^{\text {rd }}$ VEGA qualification flight data (i.e. trajectory and controller) is used in addition to the new VEGACONTROL simulator. The main goal was to analyze the new controller and mission, but also to evaluate the flexibility and versatility of the developed methodology and WCAT for changes in the system. Note that this is important as typically during the design process, especially for launchers, the mission and controller change very frequently. 
The WCAT optimization problem and methodology is cursorily presented next. The goal is to minimize a cost function (capturing a performance or robustness criterion) subject to normalized uncertainty. This optimization problem is posed as:

$$
\begin{gathered}
\min C_{\#} \\
\text { s.t. }-1<\Delta<1
\end{gathered}
$$

where $C_{\#}=\left|i_{\text {ref }}-i_{\Delta}\right|$. The cost function $C_{\#}$ is defined based on $i_{\text {ref }}$, the reference limit or nominal value of the specific performance index, and $i_{\Delta}$, the actual performance index value obtained in the presence of the uncertainty parameter set $\Delta$ used in the simulation [14]. The optimization campaigns are implemented using the Differential Evolution (DE) and Hybrid Differential Evolution (HDE) genetic algorithms, preceded by an approximate sensitivity analysis of each criterion to all and each of the variables.

The methodology adopted can be structured in the following four steps:

- Step 1 - Sensitivity analysis: This step is devoted to the analysis of the sensitivity of each cost function, by varying all the parameters around desired points, and finally selecting the variables that have the largest impact on the criteria.

- Step 2 - Worst-Case assessment: Using the previously selected variables, worst-case optimization campaigns are performed in this step.

- Step 3 - Reducing the uncertainty range: In order to devise worstcase conditions (WCCs) that are more likely to occur, the width of the interval in which the parameters can take value is reduced in this step prior to performing another WCC search (e.g. from the normalized range $[-1,1]$ to $60 \%$ of its value $[-0.6,0.6])$,.

- Step 4 - Comparison with VEGAMATH: Finally, the results obtained are assessed in VEGAMATH.

The cost functions to be used in equation (1) for worst-case launcher performance analysis are:

1. $\mathbf{C}_{1}: \mathrm{Q} \alpha$ (dynamic pressure times angle-of-attack)

2. $\mathbf{C}_{\mathbf{2}}$ : TVC A Consumption

3. $\mathbf{C}_{3}$ : TVC B Consumption

Criterion $C_{1}$ is a well-known launcher performance criteria that reflects the aerodynamic loading on the launcher, and must be ensured to be limited under a specific threshold to avoid structural damage. Criteria $C_{2}$ and $C_{3}$ measure the thrusters' consumption and thus indirectly the performance of the controller. Since the launcher is axis-symmetric and the pitch and yaw controllers are the same, except in the event of faults, failures or unexpected misalignments they are typically equivalent. Thus, a single cost function could be considered instead (namely, $C_{2}$ or $C_{3}$ or $C_{2}+C_{3}$ ) but both will be evaluated for requirement satisfaction. 
3.1 Approximate Sensitivity Analysis

This section presents a sensitivity analysis of the previous three cost functions with respect to the uncertain parameters implemented in VEGACONTROL. Although the optimization algorithms that will be used for the simulationbased worst-case search can handle large number of parameters, it is always desirable for physical understanding and insight on the results to identify a subset of the most critical uncertain parameters.

As an example, Fig. 1 shows the effect of the uncertain parameter related to combustion time (i.e. 'dTc') on a set of relevant system variables (Mach, rates, angles and aerodynamic coefficients among others). In the analysis, 'dTc' is set iteratively to $\{-1,0,1\}$ while all the remaining uncertain parameters are fixed respectively to $\{-1,0,1\}$. Thus, a total of 9 simulations are performed resulting in 3 plots as the one in Fig. 1 (one figure per each value used for the set of remaining flags). The figure shows the results for all the flags set at $\{-1\}$ and 'dTc' at $\{-1,0,1\}$. The impact of this parameter on the launcher dynamics is apparent, see for example the change with ' $\mathrm{dTc}$ ' of the $\mathrm{Q} \alpha$ response $\left(2^{\text {nd }}\right.$ row, $2^{\text {nd }}$ column from the left $)$. This which indicates that this uncertain parameter could be a main contributor to the WCCs search.
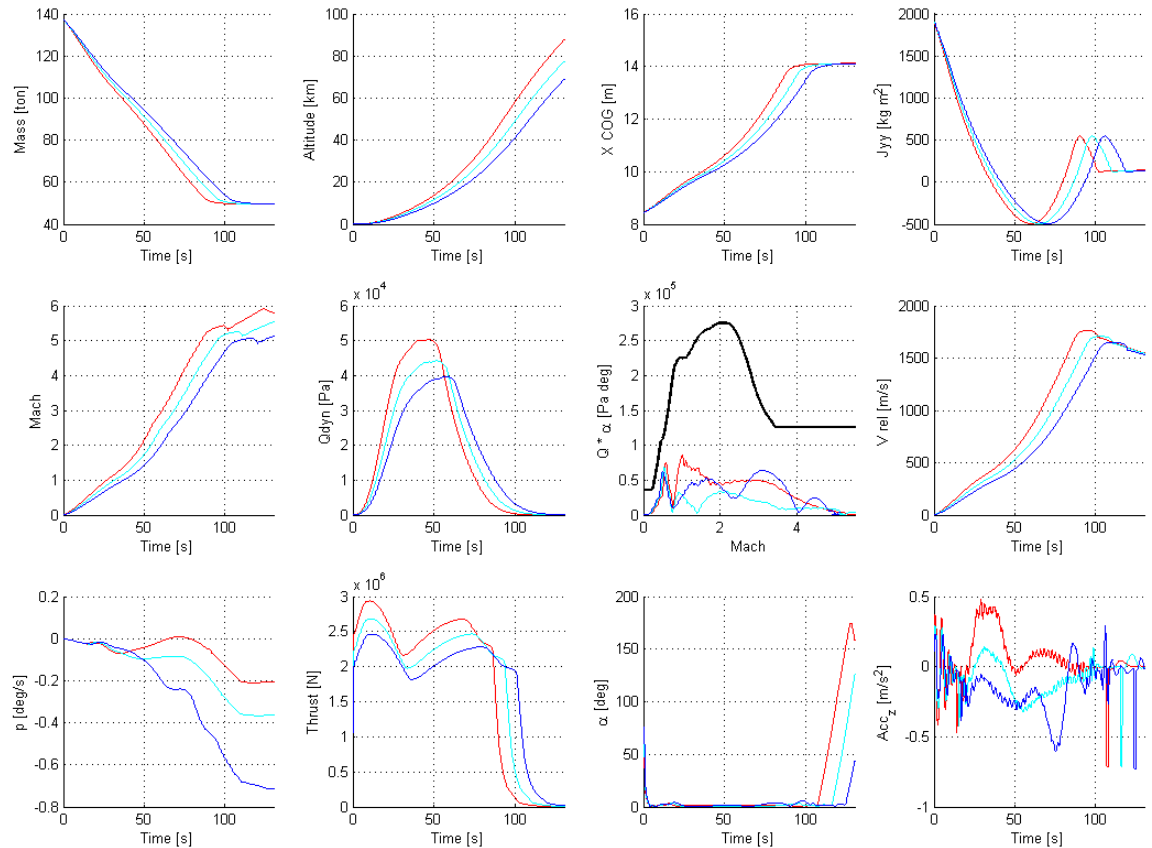

Fig. 1 Time responses of the system for 'dTc' $\{-1,0,1\}$ (red, cyan, and blue, respectively) and all other flags at $\{-1\}$ 
In order to perform the aforementioned analysis in a more systematic manner, the following algorithm was adopted enabling to compute a numerical approximation of the sensitivity of each criterion to the launcher parameters:

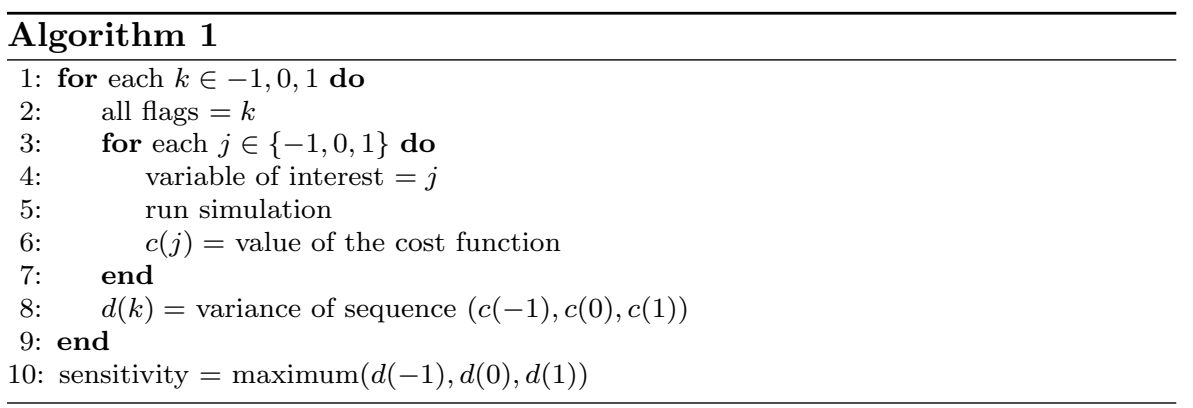

Therefore, each of the 125 variables is iteratively set at $\{-1,0,1\}$, while all other flags are set at $\{-1,0,1\}$. For each run, the values of the cost functions are calculated, while normalizing the corresponding variability with respect to the maximum value of each criterion. This leads to $125 \times 3 \times 3=1125$ simulation runs, regardless of the number of cost functions to be evaluated. This algorithm can also be illustrated by means of Fig. 2 (left), where each blue dot corresponds to a simulation run.

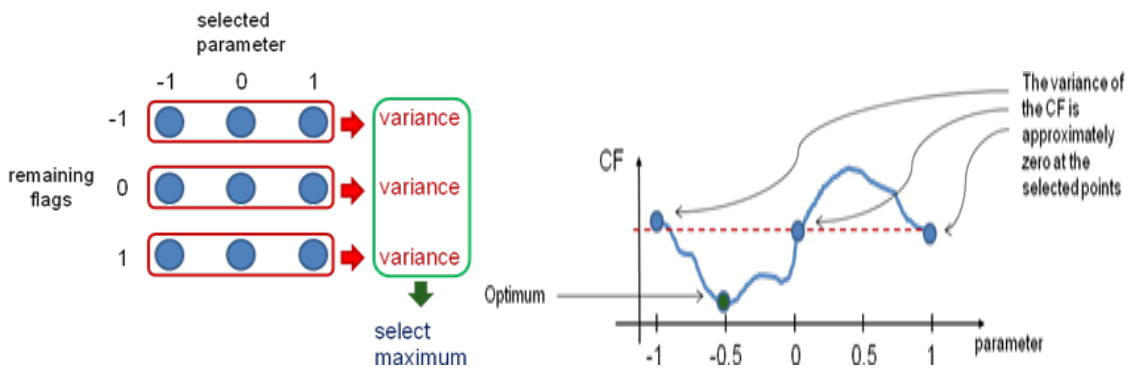

Fig. 2 Illustration of Algorithm 1 behavior (left) and example of non-convex function (right)

This sensitivity analysis of the cost functions can be extended by varying the flags within different ranges. As illustrated in Fig. 2 (right), if only the extreme values of the intervals are considered, it may not be possible to obtain an adequate approximation of the actual sensitivity of each cost function. As an example, the impact of each parameter on the 3 selected criteria is analyzed, by considering the variation of all the flags in the following three sets: $S_{1}=\{-1,0,1\} ; S_{2}=\{-0.6,0,0.6\} ;$ and $S_{3}=\{-0.3,0,0.3\}$. The results obtained are illustrated in Fig. 3 .

The sensitivity results lead to a number of conclusions in terms of the variables that should be selected, as well as the expected optimization worst-case 

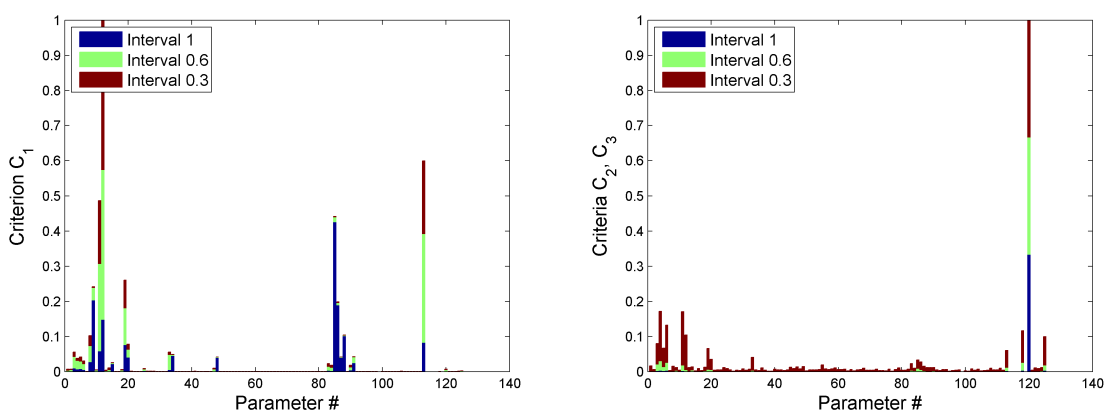

Fig. 3 Sensitivity of $C_{1}$ (left), and $C_{2}$ and $C_{3}$ (right), to all the parameters

searches to be obtained. For example, regarding the first criterion $C_{1}$, see left of Fig. 3, it can be ascertained that parameters such as \#12 ('IRSmountingZ') clearly have a significant impact for all of the intervals considered. Other parameters, such as \#85 ('TVC_bias_A') and \#86 ('TVC_bias_B'), only have a pronounced impact on $C_{1}$ if the full normalized uncertainty interval $[-1,1]$ is considered. This indicates that if a small parameter interval is used, then it is not likely that any significant benefit in terms of optimization search will be obtained from the use of these variables. Moreover, it can be seen that only about 25 out of the 125 uncertain parameters seem to have a direct impact on $C_{1}$. Thus, a considerably reduced set of variables may be selected for optimization purposes.

In terms of the TVC consumption cost functions, $C_{2}$ and $C_{3}$, the conclusion is clearly different. The uncertainty on the flexible modes frequencies, parameter \#120 ('flex_freq') has the largest impact for all of the uncertainty intervals analyzed. This makes sense from a control perspective since even small uncertainty frequency variations have a direct effect on the thruster actuation (the controller needs to quickly compensate for the offsets induced by the flexible modes in the translation and rotational movement of the thruster application point). In addition, it is well-known that flexible effects can impinge on the rigid body frequency spectrum causing undesirable effects and even instabilities. Although parameter \#120 has a critical effect, all the remaining parameters also have a non-negligible contribution -especially when the smallest range is considered. This indicates that worst-case conditions for criteria $C_{2}$ and $C_{3}$ are not likely to be obtained using a small subset of parameters.

\subsection{Selection of Worst-Case Parameter Sets}

A total of four parameter sets, formed from a selection from the 125 uncertain scatterings available in VEGACONTROL, are selected for the optimization worst-case search based on the previous analyses, see Table 1 . 
Table 1 Sets of flags to be optimized

\begin{tabular}{|c|c|}
\hline Set & Flags \\
\hline Mixed-1 & $\begin{array}{l}\text { 'dTc', 'air_density_scat', 'disp_CN', 'unc_CN', 'disp_Xcp', 'unc_Xcp', } \\
\text { 'backlash' }\end{array}$ \\
\hline Mixed-2 & 'h_wind', 'TVC_bias_A', 'TVC_bias_B', 'backlash', 'dTc', 'SRM_roll' \\
\hline Mixed-3 & $\begin{array}{l}\text { 'TVC_bias_A', 'TVC_bias_B', 'IRSmountingZ', 'SRM_roll', 'backlash', } \\
\text { 'unc_Xcp', 'flex_freq' }\end{array}$ \\
\hline Mixed-4 & $\begin{array}{l}\text { 'SRM_roll', 'backlash', 'unc_Xcp', 'flex_freq', 'air_density_scat', } \\
\text { 'P80dyCOG', 'dTc' }\end{array}$ \\
\hline
\end{tabular}

The first two sets were obtained in a previous project and using a previous version of VEGACONTROL (see [13]). They are given for comparison and completeness. The two new sets, which will be used in the WCCs search in Section 5 , are selected based on the following considerations:

- Mixed-3 contains the variables for which each of the selected criteria are more sensitive when only the interval $[-1,1]$ is considered,

- Mixed-4 contains the variables for which each of the selected criteria are more sensitive using the overall results of Fig. 3, i.e., by using intervals $[ \pm 0.3],[ \pm 0.6]$, and $[ \pm 1]$.

\section{Worst-Case $\mu$-Analysis and LFT Theory}

A concept widely used in robust control is the structured singular value $\mu$, which analytically evaluates the robustness of uncertain systems [4]. This type of robust analysis yields worst-case uncertainty combinations in a linear frequency-domain framework by using linear fractional transformation (LFT) models, which enable to embed the uncertainty into known linear operators. For the case of launchers, this translates into analyzing an LFT model numerically evaluated at different points along the flight trajectory (e.g. at a different time instances). Although a linear analysis, 25 years of worldwide use [1] validate its use for identifying WCCs valid for the nonlinear systems. Thus, for the present case, if a worst-case is found at any of these time instances then it is verified in the VEGACONTROL nonlinear simulator for the entire flight, and if it still yields a performance violation then validated in the VEGAMATH official nonlinear simulator.

In this section, the underlying theory for $\mu$ analysis and LFT modeling is cursorily reviewed. The latter is a key aspect on the application of $\mu$, since it requires a proper LFT model in order to yield meaningful results (note that by proper it is meant a model that captures the critical parametric behavior of the nonlinear system under consideration). The exact development of the LFT model will be only generally presented as the main interest of this article is on the worst-case search analysis -the interested reader is referred to $[4,1]$. 


\subsection{Structured Singular-Value Analysis}

The structured singular value $\mu_{\Delta}(M)$ of a matrix $M \in C^{n \times n}$ with respect to the uncertain matrix $\Delta$ is defined in (2), where $\mu_{\Delta}(M)=0$ if there is no $\Delta$ satisfying the determinant condition.

$$
\mu_{\Delta}(M)=\frac{1}{\min _{\Delta}(\sigma(\Delta): \operatorname{det}(I-\Delta M)=0)}
$$

Note that this definition is given in terms of an $\{M, \Delta\}$ model, which is an LFT model where $\Delta$ is typically norm-bounded $\|\Delta\|_{\infty} \leq 1$ (without loss of generality by scaling of $M$ ) for ease of calculation and interpretation. In this manner, if $\mu_{\Delta}(M) \leq 1$ then it can be guaranteed that the analyzed system, represented by the LFT, is robust to the considered uncertainty level.

The structured singular value is a robust stability $(\mathrm{RS})$ analysis but can be used also for robust performance (RP), as this problem can be transformed, in a straightforward manner, into an RS problem (see [4]).

Since $\mu_{\Delta}(M)$ is difficult to calculate exactly, the available algorithms and tools implement instead upper and lower bound calculations [1]. The upper

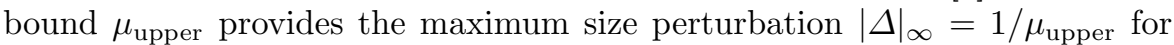
which $\mathrm{RS} / \mathrm{RP}$ is guaranteed, while the lower bound $\mu_{\text {lower }}$ guarantees the minimum size perturbation $|\Delta|_{\infty}=1 / \mu_{\text {lower }}$ for which RS/RP is guaranteed to be violated. Thus, if the bounds are close in magnitude, then the conservativeness in the calculation of $\mu$ will be small. Otherwise, no conclusion can be ascertained in terms of guaranteed system robustness for perturbations within $\left[1 / \mu_{\text {upper }}, 1 / \mu_{\text {lower }}\right]$.

\subsection{Linear Fractional Transformation Modeling}

An LFT is a representation of a system using two matrix operators, $M=$ [M11 M12; M21 M22] and $\Delta$, and a feedback interconnection. Matrix $M$ represents the nominal (known) part of the system, while $\Delta$ contains the unknown, time-varying or uncertain parameters $\rho_{i}$. Note that depending on the feedback interconnection used, there are two possible types of LFTs, lower and upper (see Fig. 4), but that both are equivalent.
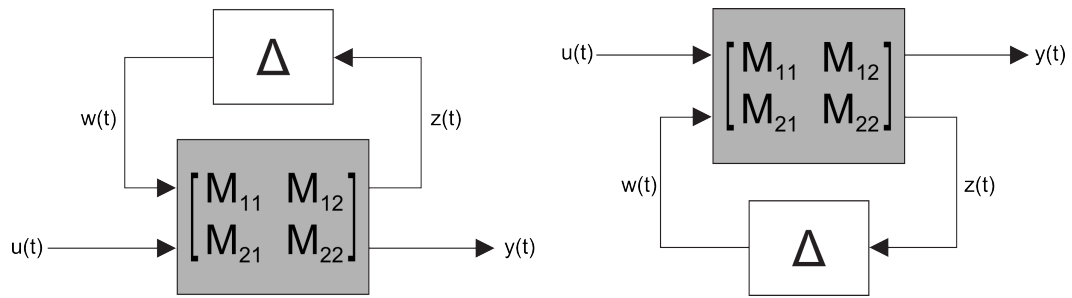

Fig. 4 Lower and upper LFTs 
Matrix $\Delta$ is unrestricted in form (structured or unstructured) and type (nonlinear, time-varying or constant), although the majority of the robust analysis, and tools, focus on real parametric static uncertainty (e.g. uncertainty in mass and inertia). It is important to note that unstructured uncertainty at component level becomes structured at system level, and that the selection of the uncertainty set $\rho(t) \in \Delta$ that captures the behavior of the nonlinear system is a task that is not always obvious a priori. Indeed, this step is key to obtain a proper LFT that will yield relevant and meaningful results and, despite its apparent simplicity, is where most of the LFT modeling effort and ingenuity is focused.

There are several approaches that can be used to obtain a proper LFT model (see $[4,9,1,10,11,12]$ and references therein). The specific approach used in the RFCS project is based on the developments from the last two references, which formalized a modeling methodology to transform a general linear parameter-varying model into an LFT representation through the use of symbolic manipulations.

\subsection{From Equations of Motion to LFT}

For any launcher vehicle, its rigid-body motion dynamics are completely described by its attitude (yaw $\psi$ or pitch $\theta$ ) and linear motion $(z$ or $y$ ) in a frame linked to the velocity of the reference trajectory. Under the assumption of an axial-symmetric launch vehicle, the dynamic equations can be expressed in state-space format as follows (the coefficients are given in Eq. (4)):

$$
\begin{array}{cc}
A=\left[\begin{array}{cccc}
0 & 1 & 0 & 0 \\
0 & a_{1} & a_{2} & a_{3} \\
0 & 0 & 0 & 1 \\
0 & a_{4} & A_{6} & a_{5}
\end{array}\right] & B=\left[\begin{array}{c}
0 \\
a_{p} \\
0 \\
K_{1}
\end{array}\right] \\
C=\left[\begin{array}{cccc}
0 & 0 & 1 & 0 \\
0 & 1 & 0 & -a_{Z} \\
1 & 0 & -a_{Z} & 0
\end{array}\right] \quad D=\left[\begin{array}{l}
0 \\
0 \\
0
\end{array}\right]
\end{array}
$$

Considering the yaw rigid-motion, the model has four states $(z, \dot{z}, \psi, \dot{\psi})$, three outputs $\left(\Delta_{\psi}, \dot{z}, z\right)$ and one input (nozzle deflection angle), and results in the following matrix coefficients:

$$
\begin{aligned}
& L_{\alpha}=S_{\mathrm{ref}} q_{\mathrm{dyn}} C_{N} \frac{1}{\alpha} ; \quad \mu_{\alpha}=-L_{\alpha}\left(x_{\mathrm{CP}}-x_{\mathrm{CoG}}\right) \frac{1}{J_{y y}} ; \quad a_{1}=-L_{\alpha} \frac{1}{m} \frac{1}{V_{\mathrm{rel}}} \\
& a_{2}=-a_{1}\left(x_{\mathrm{CP}}-x_{\mathrm{CoG}}\right) ; \quad a_{4}=-\mu_{a} \frac{1}{V_{\mathrm{rel}}} ; \quad a_{5}=-a_{4}\left(x_{\mathrm{CP}}-x_{\mathrm{CoG}}\right) ; \\
& a_{Z}=x_{\mathrm{INS}}-x_{\mathrm{CoG}} ; \quad a_{P}=-T_{h} \frac{1}{m} ; \quad a_{3}=-a c c+a_{1} V_{\mathrm{rel}} ; \\
& A_{6}=a_{4} V_{\mathrm{rel}} ; \quad K_{1}=-T_{h}\left(x_{\mathrm{CoG}}-x_{\mathrm{PVP}_{\mathrm{ref}}}\right) \frac{1}{J_{y y}} ;
\end{aligned}
$$


The launcher variables are defined as: relative velocity $V_{\text {rel }}$, dynamic pressure $q_{\mathrm{dyn}}$, thrust $T_{h}$, total launch vehicle mass $m$, total y-axis moment of inertia $J_{y y}$, normal aerocoefficient $C_{N}$, longitudinal acceleration $a c c$, angle of attack $\alpha, \mathrm{x}$-coordinate center of pressure $x_{\mathrm{CP}}$ and x-coordinate center of gravity $x_{\mathrm{CoG}}$. All these variables are dependent on time $t$ or non-gravitational speed $V N G$. The launcher dynamic model given by (3)-(4) captures the main characteristics of the $1^{\text {st }}$ atmospheric phase (P80) and is typically used to design the launchers' controllers for this phase.

The applicability of the robust $\mu$ analysis is limited by the number, repetition and type of uncertain parameters used. Thus, sensitivity analysis as those shown in Section 3.1 as well as understanding of the system physical behavior is fundamental to reduce the number of uncertain parameters. For the present case, the attention for the analytical worst-case analyses was restricted to the rigid motion (i.e. no bending nor sloshing effects). This decision was based on the need to incrementally demonstrate to ELV the validity of the $\mu+\mathrm{LFT}$ approach. Nonetheless, despite not considering flexible/sloshing effects, the worst-case obtained can be compared to those from the simulationbased WCC search since the sets of uncertain parameters used for each approach are similar.

Thus, after careful assessment of the variables in the previous matrix coefficients as well as of the scatterings (i.e. 'flags') found in the VEGACONTROL simulator, several nonlinear simulations in the same vein as those in Section 3.1 were performed to determine the most representative set of LFT uncertain parameters. The final selected parametric set is given by 6 scatterings 'flags' associated to four of the system variables from Eq. (4), see respectively $\Delta_{L F T}$ and $\Omega_{\text {sys }}$ in Eq. (5). The four time-varying variables in $\Omega_{\text {sys }}$ are introduced in the LFT using linear and bilinear polynomial fits based on the six uncertain parameters from $\Delta_{L F T}$ (which account for burning time, density scattering and dispersions disp and uncertainty unc for the normal aerodynamic coefficient and center of pressure $\mathrm{x}$-coordinate) plus symbolic nominal and deviation constants (assigned to the matrix $M$ of the LFT).

$$
\begin{aligned}
& \Delta_{L F T}=\left\{d T c, \rho_{s c a t}, \operatorname{disp}_{C N}, u_{n c_{C N}}, \operatorname{disp}_{X c p}, u n c_{X c p}\right\} \\
& \Omega_{\text {sys }}=\left\{T_{h}, \rho, C_{N}, x_{C P}\right\}
\end{aligned}
$$

The final order of the LFT is 31 and it is an exact symbolic representation of the system in (3)-(4). Also note that with respect to Table 1, the uncertainty set used for the LFT model corresponds to set Mixed-1 (but without the 'backlash' scattering since no detailed model of the thrust vector control actuator is used for the LFT model).

\subsection{Analysis Process}

The robust analysis process followed based on the developed LFT model and the $\mu$ tool is as follows: 
1. Starting from the symbolic LFT model, select the time along the P80 ascent trajectory at which the analysis is to be performed.

2. This will result in a specific $V N G$ at which the symbolic constants in the LFT matrix $M$ are numerically evaluated. Subsequently, this numerical LFT can be model reduced to obtain an LFT order of 22 . Note that except for this latter numerical model reduction, the symbolic LFT is fixed and applicable to any launcher in atmospheric phase. Also, the numerical LFT order-reduction techniques do not change the fidelity of the LFT as they mostly remove unobservable and uncontrollable rows and columns in the LFT $M$ matrix.

3. The numerically evaluated and reduced-order LFT model is closed in feedback form with the TVC controller + actuator + delay loop. The appropriate TVC controller is obtained based on data from ELV and depends on the specific $V N G$ value.

4. Select the proper $\mu$ structure for the $\Delta$ matrix. The structure can be real, complex or a mix of both [1] depending on the nature of the uncertain parameters. The advantage is that more efficient algorithms can be used for the last two types. Also in this step, the RS or RP formulation is defined. The difference is that the performance input/output channels for the $\mathrm{RP}$ case are closed using a fictitious uncertainty structure of appropriate dimensions, so that an RS problem is obtained.

5. Apply the $\mu$ analysis algorithm. This is straightforward based on the previous steps.

6. Examine and verify the results. This step serves to ensure that the robust $\mu$ upper and lower bounds are close but also to determine the validity of the perturbation (i.e. of acceptable size, nature and that results in actual violation in VEGACONTROL).

7. The results are validated by ELV using the official VEGAMATH simulator.

\section{Results}

This section describes the results obtained with both methodologies adopted in this work, i.e., simulation-based WCCs search and $\mu$-based linear analysis.

\subsection{Simulation-based WCC Search Results}

Taking into account the results obtained in [14], the Hybrid Differential Evolution (HDE) worst-case optimization algorithm was considered, as this is the technique available in the WCAT toolbox leading to the best results, for the present case.

Based on the analyses in Section 3, the selected WCC optimization campaigns are:

- Number of iterations: up to 2500 ;

- Parameter sets: Mixed-1/2/3/4; 
- Cost-functions: $C_{1}, C_{2}, C_{3}$.

Each of the 4 parameter sets is optimized separately according to each of the 3 cost-functions. Moreover, each optimization is performed by setting all the remaining flags respectively to $\{-1,0,+1\}$.

Since each iterations requires approximately 3.3 seconds in a computer with an Intel Core i5 CPU @ $3.20 \mathrm{GHz}$, the total time required for the full campaign is: $2500 \times 3 \times 3 \times 4 \times(3.3 / 3600) \approx 82.5$ hours. Alternatively, each criterion may be computed in a different Matlab session or by using Matlab's Parallel Computing Toolbox, which may significantly speed up the optimization process. The results obtained are exemplified in Table 2 using the $Q \alpha\left(C_{1}\right)$ cost-function (note that a value smaller than 1 indicates violation of this criterion). It can be observed that clear violations of this criterion were detected, regardless of the set of optimization parameters considered.

Table 2 Results for 2500 iterations, $Q \alpha$, HDE. A value smaller than 1 indicates violation of the criterion

\begin{tabular}{|c|c|c|c|}
\hline \multirow{2}{*}{ Set } & \multicolumn{3}{|c|}{ Rest of flags } \\
\cline { 2 - 4 } & $\mathbf{- 1}$ & $\mathbf{0}$ & $\mathbf{1}$ \\
\hline Mixed-1 & 0.7852 & 0.7662 & 0.6239 \\
\hline Mixed-2 & 0.9073 & 0.9298 & 0.7822 \\
\hline Mixed-3 & 0.8847 & 0.9345 & 0.8363 \\
\hline Mixed-4 & 0.6401 & 0.8033 & 0.6756 \\
\hline
\end{tabular}

For the TVC A consumption (cost-function $C_{2}$ ), the results obtained are summed up in Table 3. The results for the TVC B $\left(C_{3}\right)$ consumption are omitted, as they follow very closely those of TVC A and for ease of presentation. Unlike the $Q \alpha$ criterion, for the TVC consumption, no violations were detected.

Table 3 Results for 2500 iterations, TVC A Consumption, HDE

\begin{tabular}{|c|c|c|c|}
\hline \multirow{2}{*}{ Set } & \multicolumn{3}{|c|}{ Rest of flags } \\
\cline { 2 - 4 } & $\mathbf{- 1}$ & $\mathbf{0}$ & $\mathbf{1}$ \\
\hline Mixed-1 & 1.7420 & 1.7544 & 1.7889 \\
\hline Mixed-2 & 1.7736 & 1.7866 & 1.7991 \\
\hline Mixed-3 & 1.7788 & 1.8015 & 1.7790 \\
\hline Mixed-4 & 1.7059 & 1.7600 & 1.7391 \\
\hline
\end{tabular}

The simulation results in VEGACONTROL of the corresponding worstcases obtained by optimizing $C_{1}$ and $C_{2}$, respectively, are illustrated in Fig. 5 (first and second columns respectively). The violation of $Q \alpha$ is apparent from the plot in the upper left corner of Fig. 6 while no violation of the TVC consumptions was observed (second and third rows) even for the specific case of $C_{2}$. 

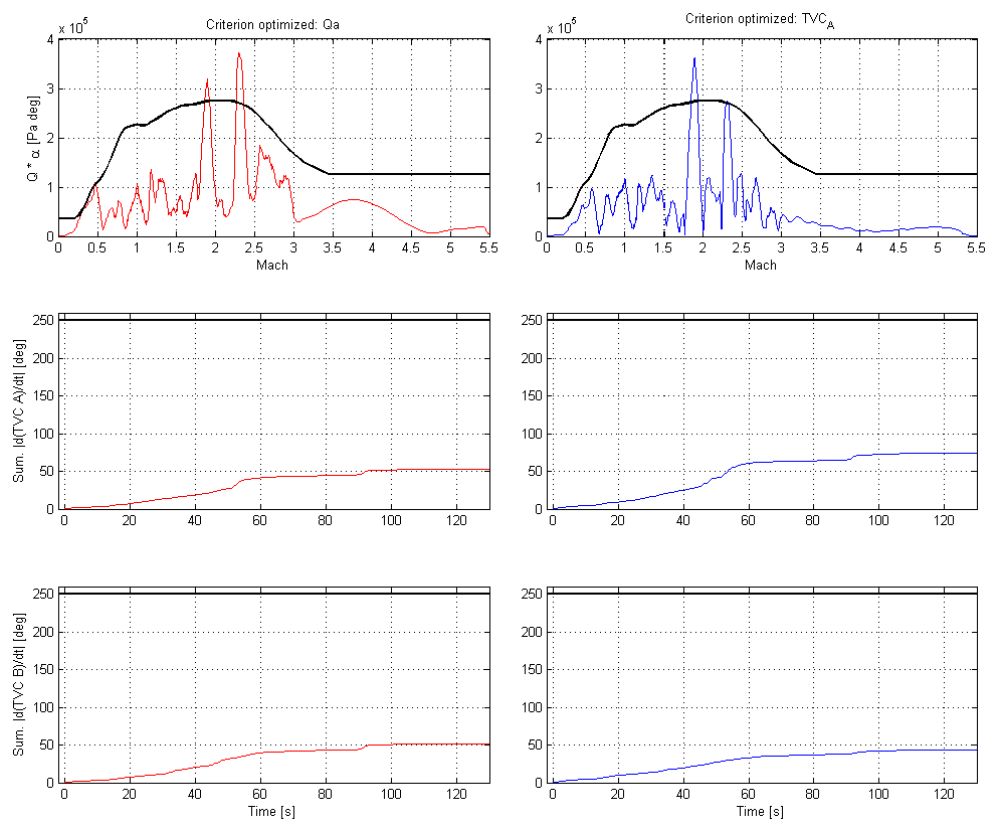

Fig. 5 Worst-cases obtained by optimizing $Q \alpha$ and the TVC consumption. The bold black lines indicate the admissible values for each criterion

\subsubsection{Uncertainty Range Reduction}

In this subsection, the width of the allowable range of variables is reduced, in order to obtain WCCs that are more likely to occur in practice. In general, having parameters at the extremes of the interval $[-1,1]$ is a low probability event, especially if one assumes a unimodal probability distribution of the parameters. The results obtained are summarized in Table 5.1.1, for the cases where the parameters are allowed to range in $\{-1,0,1\}$ and are fixed to $\{0\}$, respectively. As in the previous scenario, only violations of the $Q \alpha$ criterion are detected. Sets Mixed-1 and Mixed-4 are the ones that enable the detection of the worst-case conditions. As expected, reducing the uncertainty range also diminishes the severity of the WCC.

Table 4 Uncertainty range reduction results with different values of the remaining flags

\begin{tabular}{|c|c|c|c|}
\hline Criteria & {$[-1,1]$} & {$[-0.66,0.66]$} & {$[-0.6,0.6]$} \\
\hline \multicolumn{4}{|c|}{ All uncertain parameters at respectively $\{-1,0,1\}$} \\
\hline$Q \alpha$ & 0.6223 (Mixed-1) & 0.8414 (Mixed-4) & 0.8677 (Mixed-4) \\
\hline TVC A/B & 1.5179 (Mixed-4) & 1.7930 (Mixed-1) & 1.7720 (Mixed-4) \\
\hline \multicolumn{4}{|c|}{ All other uncertain parameters at $\{0\}$} \\
\hline$Q \alpha$ & 0.7667 (Mixed-1) & 0.8963 (Mixed-1) & 0.9224 (Mixed-1) \\
\hline TVC A/B & 1.7545 (Mixed-1) & 1.7930 (Mixed-1) & 1.7962 (Mixed-1) \\
\hline
\end{tabular}




\subsubsection{Comparison with VEGAMATH}

The WCCs obtained using WCAT+VEGACONTROL were evaluated in VEGAMATH by ELV. This allowed assessing the validity of the obtained results as well as serving as way to measure the representativeness of the two simulators. The outcomes of the simulation runs are depicted in Fig. 7, allowing the optimization parameters to vary in the interval $[-1,1]$.
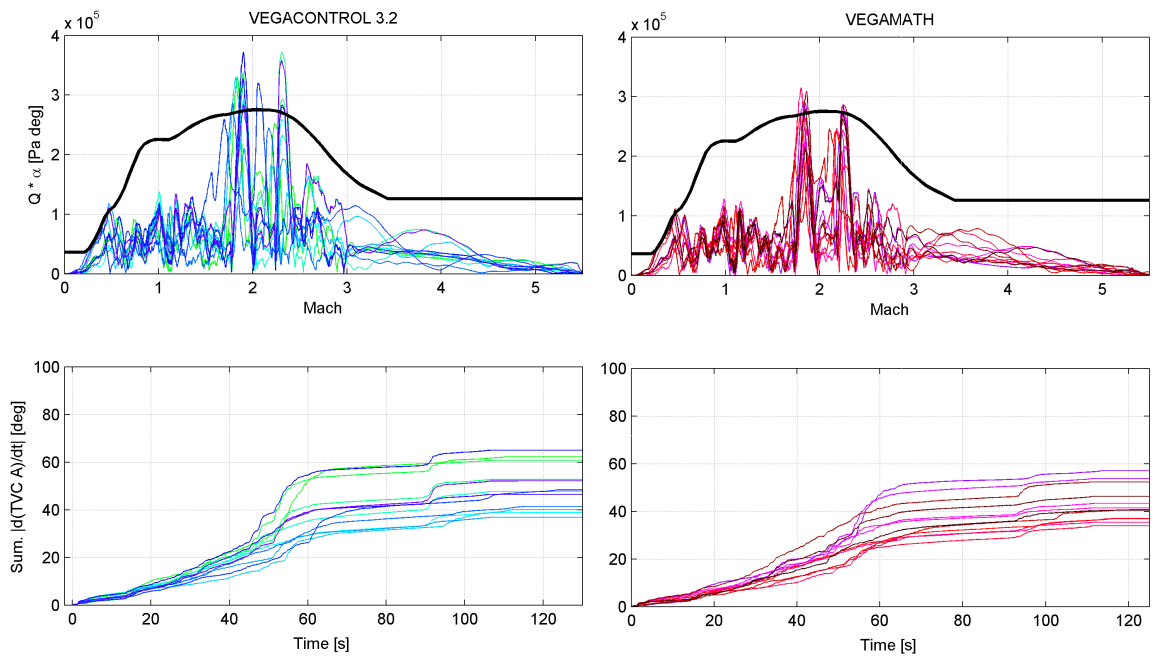

Fig. 6 Simulation of the $Q \alpha$ WCCs obtained with VEGACONTROL versus VEGAMATH

Several WCCs violating the $Q \alpha$ assumption were detected in VEGAMATH. In terms of TVC consumption, however, no violations were identified. It is also stressed that maximizing the TVC consumption leads to WCCs that, in some circumstances, violate the $Q \alpha$ criterion. This indicates that the two criteria may be aligned, as expected. Indeed, for conditions in the extremes of the flight envelope, the vehicle is likely to require an increased control effort.

\subsection{Mu Analysis WCCs Search Results}

In this section, the application of the analytical worst-case search approach known as the structured singular value $(\mu)$ to the VEGA P80 phase is presented. Several types of robust analyses can be performed with $\mu$ depending on the specific formulation of the analysis and the type of uncertainty used. In this project the focus was on robust stability (RS) and robust performance (RP) tests with real but also complex uncertainties. The latter is obtained by adding $1 \%$ complex uncertainty to each parameter in the LFT uncertainty set. 
This "complexification" of the problem helps improve the speed and convergence of the $\mu$ algorithm by avoiding numerical algorithmic issues related to pure real parameters [1].

Recall that the robust analyses performed with $\mu$ require an uncertain LFT model, and that the developed model is based on the yaw rigid-motion of VEGA during the $1^{\text {st }}$ atmospheric phase, see Section 4.3. This symbolic LFT model has 6 uncertainty parameters and a total dimension of 31 (counting the repetitions of the parameters). Prior to the application of the RS and RP $\mu$ analyses, the LFT model is used to derive two other LFT sub-models, each with a slightly different analysis purpose.

The first LFT sub-model is called perf-LFT, and is oriented towards performance analysis. Thus, it considers the output vector of the launcher as the performance channel on which to perform the analysis. The other model is called classtab-LFT and is used for evaluating classical LTI metrics such as gain and phase margins (and thus, it is a model more oriented towards stability analysis). The use of these two models allows to build up confidence on the $\mu$ analysis results, as well as serving to demonstrate the flexibility of performing different analysis starting with a main symbolic LFT model.

Using either of these two models, the $\mu$ analysis process outlined before in Section 4.4 can be followed. The first step is to numerically evaluate the corresponding LFT by choosing a time instance in the P80 flight (typically every second from 1 to 120 seconds of flight time is chosen) and fixing at a constant uncertainty level all other uncertainties not considered in the LFT (known from now on as the emphother-flags set). The data is obtained by running the VEGACONTROL simulator at the chosen uncertainty level and then extracting the data from the generated outputs at the corresponding time instance. Note, that in doing this, the flexible as well as other effects are indirectly introduced in the LFT model.

The section is divided into three subsections, the first two presenting the $\mu$ analysis results for each of the two LFT sub-models and then concluding with a comparison of the results.

\subsubsection{P80 yaw-axis performance analysis}

For the perf-LFT model, seven campaigns are performed based on seven different levels of uncertainty applied to the other-flags set. This parallels the approach used in the simulation-based WCC search approach from the previous section. The first campaign considers the nominal case (other-flags $=0)$ and the remaining six reduce the range of the uncertainty by $\{ \pm 50, \pm 30, \pm 10\} \%$.

As an example of the results, Fig. 7 shows the $\mu$-bounds corresponding to two time instances, 3 and 108 seconds. It is noted that these time instants are selected for graphical convenience (i.e. ease of visualization of the $\mu$ bounds), but might be considered, at first impression, not the most adequate for worstcase analysis. Indeed, the case at time 3 seconds is still in the vertical flight where the velocity is very low, and the case for 108 seconds is at the end of propulsion where the thrust is much decreased. 
Looking at Fig. 7, on the left-column, the real RS and RP results are given, while on the right one, the complex RS are shown (but including the RS-real upper and lower bounds to facilitate the comparison).
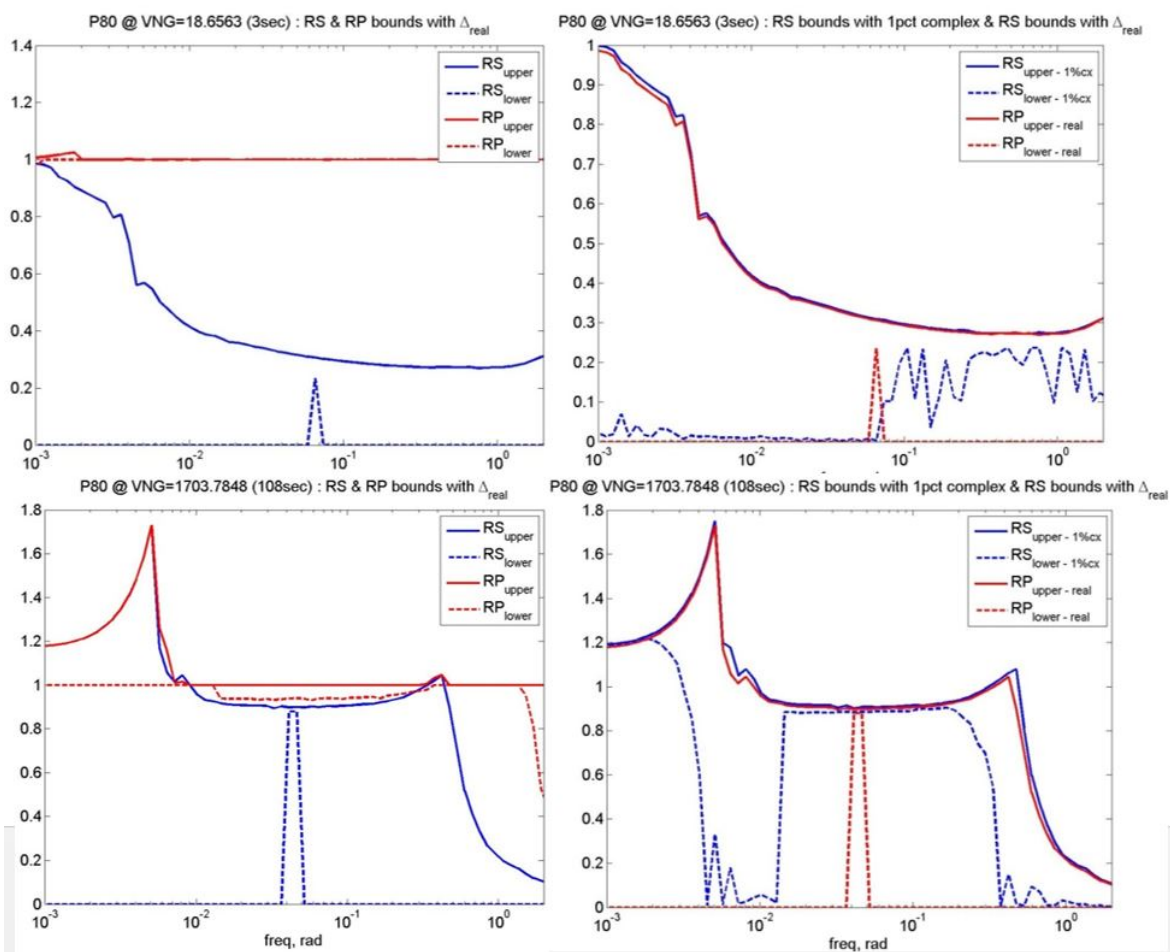

Fig. 7 VEGA P80: $\mu_{R S}$ (rob.stability) and $\mu_{R P}$ (rob.performance) bounds for perf-LFT with other-flags $=0$ : at [times 3 and 108 seconds (top \& bottom rows)] and [real- $\Delta$ and $1 \%$ complex- $\Delta$ (left \& right columns)]

Note that for the real-RP test at time 3 seconds (top-left plot), both $\mu$ bounds reach almost exactly the normalized analysis level $\mu=1$. On the other hand, for time 108 seconds (bottom-left plot) both upper bounds for the $\mu$-real $\mathrm{RP}$ and RS tests result in violations $(\mu>1)$ around the frequencies $10^{-2.5}$ and $10^{-0.5}$ radians/seconds. Since the lower-bounds for the real-RP test reach the $\mu=1$ level for both time instants, then it is expected that the worst-case effect is not due to an exogenous and temporal factor captured in the numerical data used, such as wind effects. Thus, it is expected that the worst-case obtained are the result of uncertainty effects on the system, and will carry over to the nonlinear, time-domain simulation. In addition, after further examination, it was observed that if both the upper bounds for the $\mu$-real RS and RP result in $\mu \geq 1$ level, then it is very likely that one of the perturbations will result in a $Q \alpha$ performance time-domain violation. With respect to the complex case, 
since all the bounds are below $\mu=1$ for the time instance at 3 seconds it is expected that the corresponding WCC (once turned into a real set) will not result in a violation in VEGACONTROL. On the other hand, for the time instance at 108 seconds, all the obtained WCCs could result in performance violations since they satisfy $\mu>1$.

Fig. 8 shows the $(Q \alpha$-vs-Mach) time responses for the WCCs obtained from the $\mu$-analyses shown in the previous figure. Notice that the real-RP perturbation at time 3 and the real-RS at time 108 result in violation of the time-domain bound (the solid black line). Moreover, the real-RP WCC at time 108 does not result in a violation nor do the other perturbations at 108 seconds violate the performance limit (which is in opposition to the expectation from the previous analysis of the $\mu$ bounds results).
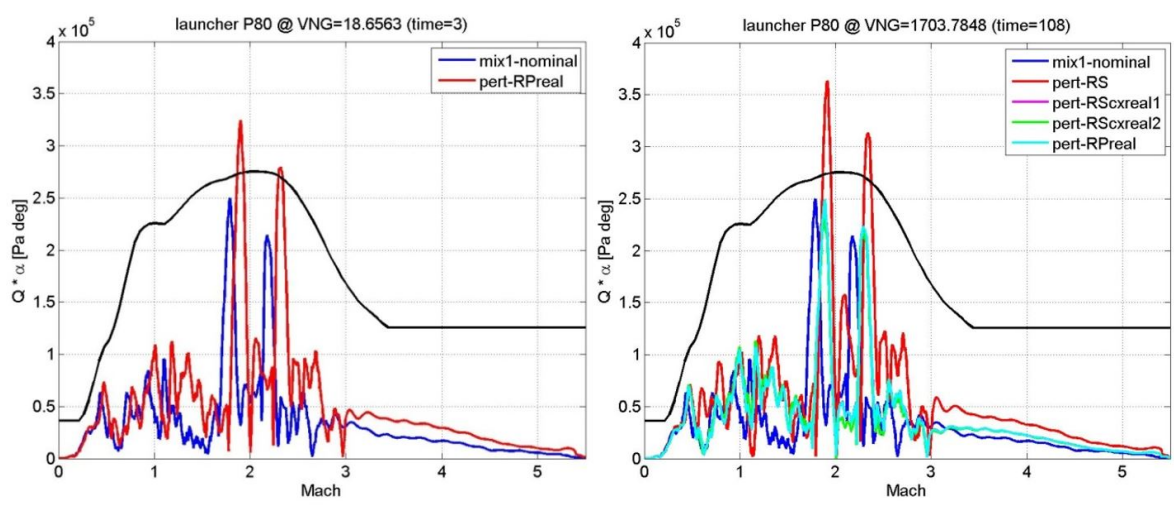

Fig. 8 VEGACONTROL P80: $Q \alpha$ responses to $\mu_{W C}$ obtained at time $\{3,108\}$ seconds

It is highlighted that this disparity between the frequency-domain and the time-domain results arises from two sources: (i) the frequency versus time domains analysis, and more importantly (ii) the time-varying nature of the nonlinear system in the time-domain analysis. With respect to the latter issue, note that the frequency analysis is performed in a linear-time-invariant frame and at a single time instant (which might be far away from, or even after the time at, which the violation occurs in the time simulation). Nonetheless, it is seen that, despite these differences, WCCs are found using the proposed $\mu$ methodology that result in violations in VEGACONTROL.

\subsubsection{P80 yaw-axis Gain/Phase MARGIN analysis}

In this subsection the $\mu$-analysis is used to search for WCCs based on classical gain and phase margin $(\mathrm{GM} / \mathrm{PM})$ violations. The process to obtain the classtab-LFT sub-model starting from the general symbolic LFT from Section 4.3 , is just a matter of including a multiplicative uncertainty related to an ellipse in the Nyquist plane, see Eq. (6). This ellipse is then introduced at 
the input of the P80 yaw-axis model (i.e. the TVC command). The resulting $\mathrm{GM} / \mathrm{PM}$ uncertainty is represented by a single additional uncertain parameter $\delta_{G P M}$ included in the $\Delta_{L F T}$ set -plus two additional symbolic constants, $a$ and $W_{n}=-r / a$, which are placed in the LFT $M$ matrix. The symbolic constants are related to the center $(-a)$ and radius $(r)$ of the Nyquist plane ellipse defined by the GM/PM requirements [7]. Thus, by assigning a numerical value to the required GM and PM, a two-equation system can be solved to yield the corresponding values for $a$ and $r$ (and thus $W_{n}$ ). In the present case, the GM and PM limits for the nominal case (other-flags=0) are $3 \mathrm{~dB}$ and 43 degrees respectively, and arise from the VEGA requirements.

$$
\begin{gathered}
P=P_{0} a\left(1+W_{n} \delta_{G P M}\right) \\
G M=20 \log _{10}(a+r) \\
P M=\cos ^{-1}\left(\frac{a^{2}+r^{2}+1}{2 a}\right)
\end{gathered}
$$

Fig. 9 is the equivalent to Fig. 7, but for the classtab-LFT sub-model and only at time instant 3 seconds. It is clearly seen, in comparison to Fig. 7, that for the classtab- LFT model, the obtained real-RS bounds rise to the normalized analysis level $\mu=1$, indicating a likely violation in the time-domain by the corresponding WCC. This is examined further in the next sub-sections when comparing the results from both models.
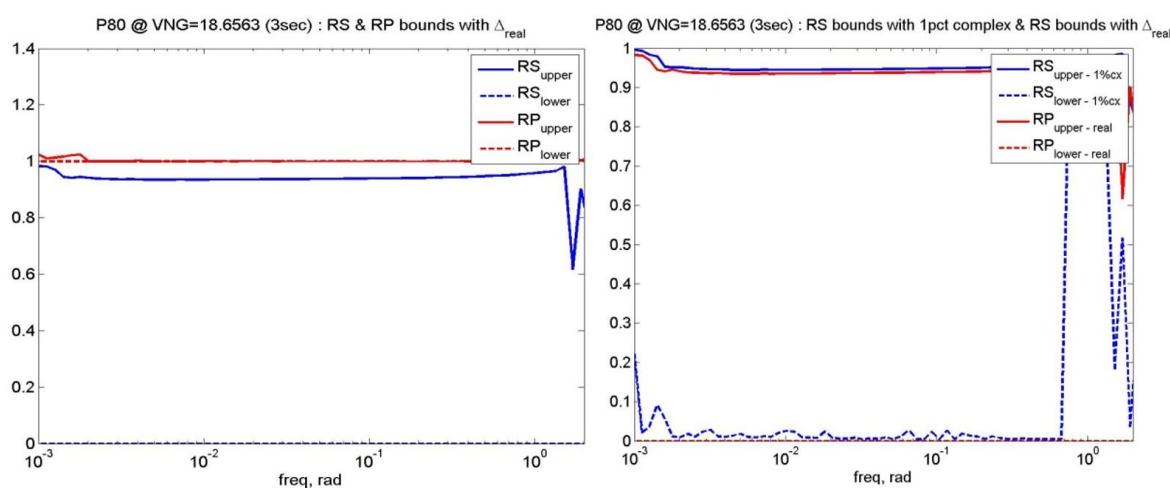

Fig. 9 VEGA P80: $\mu_{R S}$ and $\mu_{R P}$ with classtab-LFT at $[$ other-flags $=0$, time $=3$ seconds $]$ for real- $\Delta$ (left column) and $1 \%$ complex- $\Delta$ (right column)

\subsubsection{Comparison of the results between perf-LFT and classtab-LFT}

For the perf-LFT sub-model, the results indicate that the followed analytical worst-case algorithm and process can successfully identify many combinations that result in a violation, or even strong violation, in the time-domain of 
the $Q \alpha$-versus-Mach limit (which is set to ensure structural integrity of the launcher). Furthermore, these WCCs are found well spread out through the range of the uncertainty LFT set and, indeed, by examining their spread, several violation-regions can be identified, see 10 . This latter remark is important as it shows that these worst-cases are not improbable since there are regions of violation and thus the probability of encountering them is not negligible. Nonetheless, it is noted that no quantifiable probability can be associated to this conclusion.

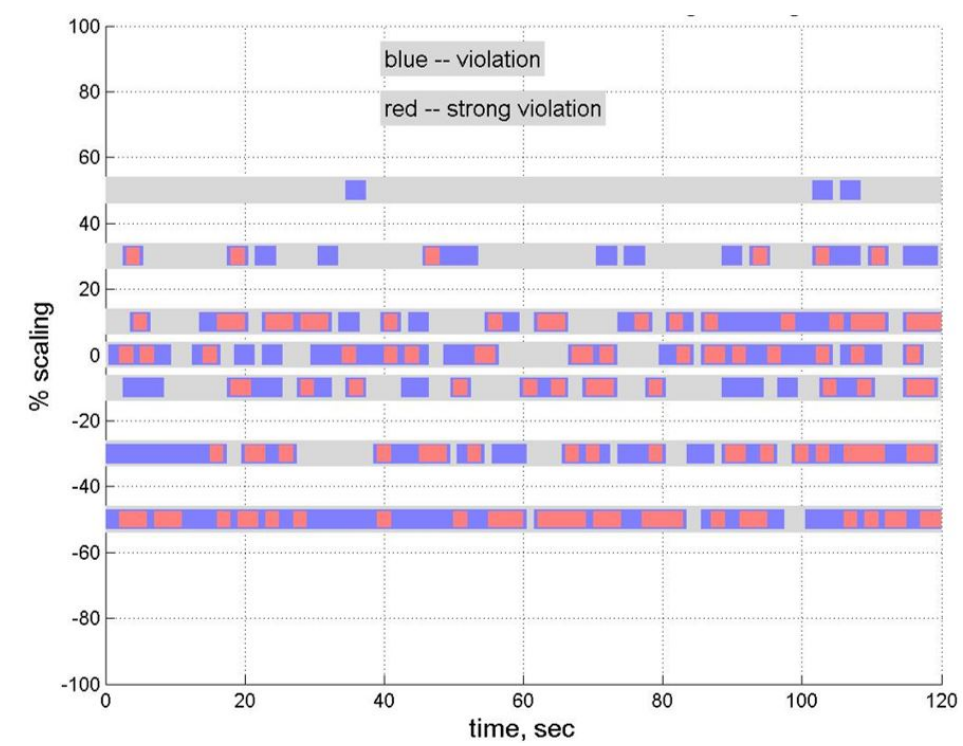

Fig. 10 VEGA P80: $Q \alpha$ violations (time instance versus \% scaling for other-flags set)

With respect to the classtab-LFT sub-model, although the previous LFT model is better at obtaining strong performance violations the use of the classtab LFT model results in many more non-strong violations. This is the case since the former model focuses on time-domain performance violation while the latter model is geared more specifically towards LTI stability violations. And, although a strong violation of stability will likely result in a strong performance violation, for launchers (and specially VEGA) the linear stability margin requirements are relatively benign due to the fast dynamics of the system (i.e. there is no time for build up). In addition, the classtab LFT model has the advantage of providing the worst-case (low and high frequency) gain and phase margins directly, which can be easily visualized against the time instance used for the analysis, see Fig. 11. Finallly, note that the WCCs obtained with the two LFT models are different but all show a general trend from which several different WC combinations can be easily obtained. 

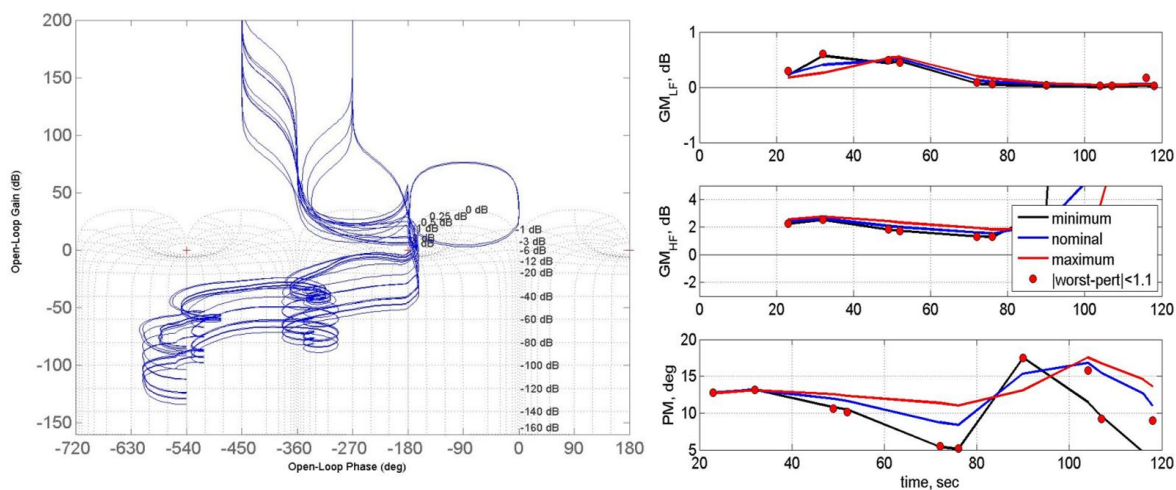

Fig. 11 VEGA P80: Nichols (left) and GM/PM (left) non-strong viol. [other-flags $=+30 \%$ ]

Fig. 12 shows the VEGACONTROL $Q \alpha$-vs-Mach "strong" violations (i.e. those where $Q \alpha \geq 3 \times 10^{5} \mathrm{~Pa}$ deg) corresponding to all the WCCs obtained using the perf-LFT (left column) and classtab-LFT (right column).
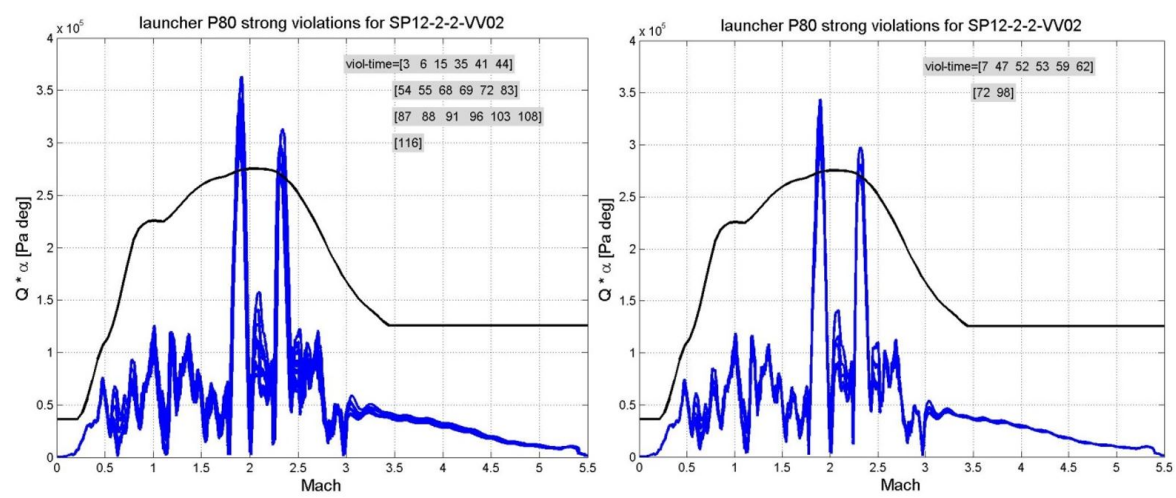

Fig. 12 VEGACONTROL P80: $Q \alpha$ for $\mu_{W C}$ from perf-LFT (left) and classtab-LFT (right)

In gray, on the right-upper corner, the time instants at which the WCCs are obtained are listed, and are given here:

$$
\begin{aligned}
& L F T_{\text {perf }}=\{3,6,15,35,41,44,54,55,68,69,72,83,87,88,91,96,103,108,116\} \\
& L F T_{\text {classtab }}=\{7,47,52,53,59,62,72,96\}
\end{aligned}
$$

It is seen that many more violations are obtained using perf-LFT than classtab-LFT: 19 instants for the first versus 8 for the other. The opposite is true for "non-strong" violations $(Q \alpha<3)$, where the classtab-LFT yields 58 instances versus 20 for the perf-LFT. Again, it is highlighted that this has to do with the fact that for the former LFT the aim is to maximize the stability 
violation which for the nonlinear, time-varying simulator model it might not have sufficient time to develop resulting in a "non-strong" violation that only at times is "strong". Similar results were obtained by ELV using VEGAMATH.

\section{Conclusions}

This paper described the methods investigated within the RFCS project to enhance the state-of-practice for launcher $\mathrm{V} \& \mathrm{~V}$, specifically for the VEGA launcher. The focus was on worst-case condition search methods and the project covered simulation-based optimization algorithms as well as linear, analytical approaches. For the first type, the methods selected considered global and hybrid (global and local) optimization algorithms applied to costs functions capturing the performance objectives of the launcher. The second type, considered linear robust analysis tools based on the structured singular value formalism together two different types of LFT models that allowed focusing on worst-cases affecting performance or linear stability.

For the first type of methods, the Worst-Case Analysis Optimization Tool (WCAT) was used and the specific optimization algorithms were Differential Evolution (DE) and Hybrid Differential Evolution (DHE). The optimization campaigns were preceded by an approximate sensitivity analysis of each criterion to all the uncertainty variables. This sensitivity analysis led to select a subset of uncertain parameters, which was done to facilitate insight and understanding on the evolution of the worst-cases and their significance. Worst-cases were obtained with both algorithms that resulted in violation of the well-known launcher $Q \alpha$ criteria. In particular, the bending modes present in the new version of VEGACONTROL resulted in an increased in the number of WCCs violating the requirements of the launch vehicle. This was also compounded by the stronger wind profiles used in this project, the effect of which is clearly seen by the number of violations around the maximum $Q \alpha$ region. Moreover, theses $Q \alpha$ violations occurred even if the uncertainty level was constrained to $\pm 60 \%$ of its normalized value, which indicates that the identified worst-cases are not improbable -a typical complaint against WCC search methods.

With respect to the analytical WCC approach, a similar sensitivity approach was followed but in this case this is a critical step as the number of uncertain parameters used can severely limit their applicability. Thus, the focus for this type of WCC search algorithm was on the yaw rigid-motion of VEGA. Nonetheless, the numerical evaluation of the resulting LFT model used for the robust analysis was performed using time response data from the VEGACONTROL simulation, and thus, contained also the effects of the wind and the flexible modes. Robust stability and performance analysis were performed using two different LFT sub-models derived from the general symbolic LFT model of the launcher. These LFT sub-models enabled to focus on searching WCCs that maximized the violation of performance objectives or linear stability (i.e. low and high frequency gain and phase) margins. Both models enabled to identified WCCs that resulted in $Q \alpha$ violations in VEGACONTROL. 
All the WCCs obtained using both type of approaches, and that resulted in violations in VEGACONTROL, were then validated by ELV, the prime contractor for VEGA and responsible for its GNC, on the official VEGA nonlinear, high-fidelity simulator VEGAMATH. The majority of these WCCs also resulted in violations in the latter, indicating that the approaches were capable of correctly identifying conditions using down-scale versions of the official simulator, and in the case of the linear $\mu$ robust analysis, a simplified linear analysis model of the launcher dynamics.

In comparison to standard Monte Carlo approaches, the present methods serve to complement them since they look at the identification of worst-cases as opposed to providing statistical guarantees on the non-violation of objectives. And they are able to do this identification at a fraction of the computational or time cost of the Monte Carlo approaches. For example, the HDE algorithm was capable of detecting worst-cases using only 2,500 simulation runs of VEGACONTROL while the $\mu$ analysis took about 30 seconds per time instance and was capable of detecting worst-cases by just looking at 120 time instances extracted from a nominal time simulation of VEGACONTROL. This compares extremely favorably with the usual tens of thousand, or even hundreds of thousand, simulation runs required in Monte Carlo to achieve significant statistic guarantees.

This work was funded by the European Space Agency under ESA-ESTEC contract 1-6322/09/NL/JK.

\section{References}

1. Gary J Balas, John C Doyle, Keith Glover, Andy Packard, and Roy Smith. $\mu$-analysis and synthesis toolbox. MUSYN Inc. and The MathWorks, Natick MA, 1998.

2. Alec Bateman, David Ward, and Gary Balas. Robust/worst-case analysis and simulation tools. In Proc. AIAA Guidance, Navigation, and Control Conference, 2005.

3. Christine M Belcastro and Celeste M Belcastro. On the validation of safety critical aircraft systems, part i: An overview of analytical \& simulation methods. In AIAA Guidance, Navigation and Control Conference, Austin, Texas, USA, volume 20, 2003.

4. John Doyle, Andy Packard, and Kemin Zhou. Review of LFTs, LMIs, and $\mu$. In Decision and Control, 1991., Proceedings of the 30th IEEE Conference on, pages 1227-1232. IEEE, 1991.

5. Christopher Fielding, Andras Varga, Samir Bennani, and Michiel Selier. Advanced techniques for clearance of flight control laws, volume 283. Springer, 2002.

6. John M Hanson and Bernard B Beard. Applying monte carlo simulation to launch vehicle design and requirements verification. Journal of Spacecraft and Rockets, 49(1):136-144, 2012. 
7. J. Hansson. Using Linear Fractional Transformations for Clearance of Flight Control Laws. MS Thesis, Linkoping University, Sweden, 2003.

8. Stephen A Jacklin, Johann M Schumann, Pramod P Gupta, R Richard, Kurt Guenther, and Fola Soares. Development of advanced verification and validation procedures and tools for the certification of learning systems in aerospace applications. In Proceedings of Infotech Aerospace Conference, Arlington, VA, 2005.

9. Paul Lambrechts, Jan Terlouw, Samir Bennani, and Maarten Steinbuch. Parametric uncertainty modeling using LFTs. In American Control Conference, 1993, pages 267-272. IEEE, 1993.

10. Jean-Francois Magni. Linear fractional representation toolbox for use with matlab. available at http://w3.onera.fr/smac/, 2006.

11. Andrés Marcos and Gary J Balas. Development of linear-parametervarying models for aircraft. Journal of Guidance, Control, and Dynamics, 27(2):218-228, 2004.

12. Andrés Marcos, Declan G Bates, and Ian Postlethwaite. A symbolic matrix decomposition algorithm for reduced order linear fractional transformation modelling. Automatica, 43(7):1211-1218, 2007.

13. Andrés Marcos, Hector Garcia De Marina, Valerio Mantini, Christophe Roux, and Samir Bennani. Optimization-based worst-case analysis of a launcher during the atmospheric ascent phase. In AIAA Guidance, Navigation, and Control Conference and Exhibit, 2013.

14. Andres Marcos, Valerio Mantini, Christophe Roux, and Samir Bennani. Bridging the gap between linear and nonlinear worst-case analysis: An application case to the atmospheric phase of the vega launcher. In IFAC Automatic Control in Aerospace, Würzburg, Germany, volume 19(1), pages 42-47, 2013.

15. Andrés Marcos, Christophe Roux, Max Rotunno, Hans-Dieter Joos, Samir Bennani, Luis F. Penín, and Augusto Caramagno. The V\&V problematic for launchers: current practice and potential advantages on the application of modern analysis techniques. In ESA Guidance, Navigation and Control Conference, Karlovy Vary, Czech Republic, 2011.

16. Prathyush P Menon, Ian Postlethwaite, Samir Bennani, Andrés Marcos, and Declan Bates. Robustness analysis of a reusable launch vehicle flight control law. Control Engineering Practice, 17(7):751 - 765, 2009.

17. Rainer Storn and Kenneth Price. Differential evolution-a simple and efficient heuristic for global optimization over continuous spaces. Journal of global optimization, 11(4):341-359, 1997. 